PROCEEDINGS OF THE

AMERICAN MATHEMATICAL SOCIETY

Volume 132, Number 3, Pages 789-798

S 0002-9939(03)07174-0

Article electronically published on August 7, 2003

\title{
THE SHARP LOWER BOUND FOR THE FIRST POSITIVE EIGENVALUE OF A SUB-LAPLACIAN ON A PSEUDO-HERMITIAN MANIFOLD
}

\author{
SONG-YING LI AND HING-SUN LUK
}

(Communicated by Mei-Chi Shaw)

\begin{abstract}
This paper studies, using the Bochner technique, a sharp lower bound of the first eigenvalue of a subelliptic Laplace operator on a strongly pseudoconvex CR manifold in terms of its pseudo-Hermitian geometry. For dimensions greater than or equal to 7 , the lower bound under a condition on the Ricci curvature and the torsion was obtained by Greenleaf. We give a proof for all dimensions greater than or equal to 5 . For dimension 3, the sharp lower bound is proved under a condition which also involves a distinguished covariant derivative of the torsion.
\end{abstract}

\section{INTRODUCTION AND MAIN RESULTS}

Let $M$ be a $(2 n+1)$-dimensional strongly pseudoconvex CR manifold and $H(M)$ the structure bundle, where $H(M)$ is a subbundle of the complexified tangent bundle $T_{\mathbb{C}}(M)$ of which each fiber is an $n$-dimensional complex vector space. Let $\theta$ be a real nonvanishing one-form on $M$ that annihilates $H(M) \oplus \overline{H(M)}$. Then, $(M, \theta)$ is a strongly pseudoconvex pseudo-Hermitian manifold in the sense of Webster [9]. Locally, one can choose $n$ complex one-forms $\theta^{\alpha}$, so that $\left(\theta, \theta^{\alpha}, \theta^{\bar{\alpha}}\right)$ form a basis of complex covectors and

$$
d \theta=i \theta^{\alpha} \wedge \theta^{\bar{\alpha}}, \quad \theta^{\bar{\alpha}}=\overline{\theta^{\alpha}} .
$$

The local coframe $\left(\theta, \theta^{\alpha}, \theta^{\bar{\alpha}}\right)$ is uniquely determined up to

$$
\theta=\theta^{\prime}, \quad \theta^{\alpha}=\theta^{\prime} U_{\beta}^{\alpha}, \quad \theta^{\bar{\alpha}}=\theta^{\prime} \bar{\beta} U_{\bar{\beta}}^{\bar{\alpha}}
$$

where

$$
U_{\beta}^{\alpha} U_{\bar{\gamma}}^{\bar{\alpha}}=\delta_{\beta \bar{\gamma}}, \quad U_{\bar{\beta}}^{\bar{\alpha}}=\overline{U_{\beta}^{\alpha}} .
$$

If we compare the dual frame

$$
X_{0}=\overline{X_{0}}, \quad X_{\alpha}, \quad X_{\bar{\alpha}}=\bar{X}_{\alpha}
$$

Received by the editors October 28, 2002.

2000 Mathematics Subject Classification. Primary 32V05, 32V20; Secondary 53C56.

Key words and phrases. Strongly pseudoconvex CR manifold, pseudo-Hermitian geometry, sub-Laplacian, eigenvalues. 
to $\left(\theta, \theta^{\alpha}, \theta^{\bar{\alpha}}\right)$, then the transformation (1.2) gives

$$
X_{0}^{\prime}=X_{0}, \quad X_{\alpha}^{\prime}=U_{\alpha}^{\beta} X_{\beta}, \quad X_{\bar{\alpha}}^{\prime}=U_{\bar{\alpha}}^{\bar{\beta}} X_{\bar{\beta}},
$$

which singles out a unique transversal $X_{0}$ to $H(M) \oplus \overline{H(M)}$. Furthermore,

$$
d \theta^{\alpha}=\theta^{\beta} \wedge w_{\beta}^{\alpha}+\theta \wedge \tau^{\alpha}
$$

where $w_{\beta}^{\alpha}$ are connection 1-forms that are skew-Hermitian:

$$
w_{\beta}^{\alpha}=-w_{\bar{\alpha}}^{\bar{\beta}}
$$

and the $\tau^{\alpha}$ are torsion 1 -forms of type $(0,1)$ :

$$
\tau^{\alpha}=A_{\overline{\alpha \gamma}} \theta^{\bar{\gamma}}, \quad A_{\overline{\alpha \gamma}}=A_{\overline{\gamma \alpha}} .
$$

Moreover, if we define curvature 2 -forms $\Omega_{\beta}^{\alpha}$ by

$$
\Omega_{\beta}^{\alpha}=d w_{\beta}^{\alpha}-w_{\beta}^{\gamma} \wedge w_{\gamma}^{\alpha}-i \theta^{\bar{\beta}} \wedge \tau^{\alpha}+i \tau^{\bar{\beta}} \wedge \theta^{\alpha},
$$

then

$$
\Omega_{\beta}^{\alpha}=R_{\beta \bar{\alpha} \rho \bar{\sigma}} \theta^{\rho} \wedge \theta^{\bar{\sigma}}+\lambda_{\beta \bar{\alpha}} \wedge \theta
$$

where $\lambda_{\beta \bar{\alpha}}$ are 1-forms and the curvature tensor components $R_{\beta \bar{\alpha} \rho \bar{\sigma}}$ satisfy

$$
R_{\beta \bar{\alpha} \rho \bar{\sigma}}=R_{\bar{\alpha} \beta \bar{\sigma} \rho}=R_{\rho \bar{\alpha} \beta \bar{\sigma}} .
$$

Let

$$
\Gamma_{\beta j}^{\alpha}=\omega_{\beta}^{\alpha}\left(X_{j}\right), \quad \Gamma_{\bar{\beta} j}^{\bar{\alpha}}=\omega \frac{\bar{\alpha}}{\beta}\left(X_{j}\right)
$$

where $\alpha, \beta \in I$ with $I=\{1, \cdots, n\}$ and $j \in\{0\} \cup I \cup \bar{I}$. Then $R_{\alpha \bar{\beta} \rho \bar{\sigma}}$ can also be written as

$$
R_{\alpha \bar{\beta} \rho \bar{\sigma}}=X_{\rho}\left(\Gamma_{\alpha \bar{\sigma}}^{\beta}\right)-X_{\bar{\sigma}}\left(\Gamma_{\alpha \rho}^{\beta}\right)+\Gamma_{\alpha \gamma}^{\beta} \Gamma_{\rho \bar{\sigma}}^{\gamma}-\Gamma_{\alpha \bar{\gamma}}^{\beta} \Gamma_{\bar{\sigma} \rho}^{\bar{\gamma}}+\Gamma_{\alpha \bar{\sigma}}^{\gamma} \Gamma_{\gamma \rho}^{\beta}-\Gamma_{\alpha \rho}^{\gamma} \Gamma_{\gamma \bar{\sigma}}^{\beta}+i \delta_{\rho \bar{\sigma}} \Gamma_{\alpha 0}^{\beta} .
$$

For $X=\sum_{\alpha=1}^{n} x^{\alpha} X_{\alpha}$ where $x^{\alpha}$ are local functions, we let

$$
\operatorname{Ric}(X, X)=R_{\alpha \bar{\beta}} x^{\alpha} \overline{x^{\beta}}, \quad R_{\alpha \bar{\beta}}=g^{\rho \bar{\sigma}} R_{\alpha \bar{\beta} \rho \bar{\sigma}}=R_{\alpha \bar{\beta} \rho \bar{\rho}},
$$

and

$$
\operatorname{Tor}(X, X)=i\left(A_{\bar{\alpha} \bar{\beta}} \overline{x^{\alpha}} \overline{x^{\beta}}-A_{\alpha \beta} x^{\alpha} x^{\beta}\right) .
$$

Then the covariant derivatives and the sub-Laplacian of a function $f$ on $M$ are given by

$$
f_{j}=X_{j} f, \quad f_{\alpha j}=X_{j} f_{\alpha}-\Gamma_{\alpha j}^{\gamma} f_{\gamma}, \quad f_{\bar{\alpha} j}=X_{j} f_{\bar{\alpha}}-\Gamma_{\bar{\alpha} j}^{\bar{\gamma}} f_{\bar{\gamma}}
$$

and

$$
\tilde{\Delta} f=2 \operatorname{Re}\left(\operatorname{tr}\left(\pi_{+} D^{2} f\right)\right)=\sum_{\alpha} f_{\alpha \bar{\alpha}}+f_{\bar{\alpha} \alpha} .
$$

Let $\lambda_{1}$ be the first positive eigenvalue of $\tilde{\Delta}$. We shall prove the following theorems.

Theorem 1.1. Let $n \geq 2$ and let $M$ be a $(2 n+1)$-dimensional strongly pseudoconvex pseudo-Hermitian manifold in the sense of Webster. If

$$
\operatorname{Ric}_{m}(X, X)+(n / 2) \operatorname{Tor}_{m}(X, X) \geq k_{0} g_{m}(X, X)
$$

for all $m \in M$ and $X \in H_{m}(M)$, for some positive constant $k_{0}$, then $\lambda_{1} \geq \frac{n k_{0}}{n+1}$. 
When $n \geq 3$, Theorem 1.1 was established and proved by A. Greenleaf in [5], where he gave a fundamental Bochner formula for the sub-Laplacian. Our contribution here is proving the theorem when $n=2$, but the argument in Section 2 works for $n \geq 2$.

For the case $n=1$, we need one more condition on the torsion term $A_{11}$. We shall prove the following theorem.

Theorem 1.2. Let $M$ be a 3-dimensional strongly pseudoconvex pseudo-Hermitian manifold in the sense of Webster. Let

$$
\operatorname{Ric}_{m}(X, X)+\frac{1}{2} \operatorname{Tor}_{m}(X, X)-\frac{3}{k_{0}} B_{m}^{2}(X, X) \geq k_{0} g_{m}(X, X),
$$

for all $m \in M, X \in H_{m}(M)$, and for some positive constant $k_{0}$, where

$$
B_{m}^{2}\left(x_{1} X_{1}, x_{1} X_{1}\right)=2\left|A_{11}\right|^{2}\left|x_{1}\right|^{2}-\operatorname{Re} X_{0}\left(A_{\overline{11}}\right) x_{1}^{2}-2 \operatorname{Re} A_{\overline{11}} \Gamma_{10}^{1} x_{1}^{2} .
$$

Then $\lambda_{1} \geq \frac{k_{0}}{2}$.

The above two theorems are sharp when $M$ is the unit sphere in $\mathbb{C}^{n+1}$, in which case the torsion vanishes and $\lambda_{1}=\frac{n}{n+1} k_{0}=n$.

Observing that $\left(A_{\overline{11}}\right)_{0}=X_{0} A_{\overline{11}}-2 A_{\overline{11}} \Gamma_{\overline{1} 0}^{\overline{1}}=X_{0} A_{\overline{11}}+2 A_{\overline{11}} \Gamma_{10}^{1}$, we remark that while the pseudo-Hermitian case differs from the Riemannian case in that torsion enters into the picture in addition to the Ricci curvature, the 3-dimensional pseudoHermitian case differs from the higher-dimensional cases in that the first covariant derivative of the torsion along the distinguished transversal $X_{0}$ also plays a role in Theorem 1.2.

\section{Proof of Theorem 1.1}

We shall start to prove Theorem 1.1.

Proof. Let $\left(X_{0}, X_{\alpha}, X_{\bar{\alpha}}\right)$ be a local frame given by (1.4). Let $X_{\alpha}^{*}$ be the adjoint of $X_{\alpha}$ with respect to $d v$. Then

$$
X_{\alpha}^{*}=-X_{\alpha}+\left(\sum_{\beta} \Gamma_{\beta \bar{\beta}}^{\alpha}\right) \text { and } \tilde{\Delta}=-\sum_{\alpha}\left(X_{\alpha}^{*} X_{\alpha}+X_{\bar{\alpha}}^{*} X_{\bar{\alpha}}\right)
$$

Let $\tilde{\nabla} f=\sum_{\alpha} f_{\bar{\alpha}} X_{\alpha} \in \Gamma(H(M))$ and $\tilde{d} f=f_{\alpha} \theta^{\alpha}+f_{\bar{\alpha}} \theta^{\bar{\alpha}}$. We recall the following formulae proved in [5].

Bochner formula:

$$
\begin{aligned}
& \frac{1}{2} \tilde{\Delta}|\tilde{\nabla} f|^{2}=\left\|\pi_{+} D^{2} f\right\|^{2}+\left\|\pi_{-} D^{2} f\right\|^{2}+\operatorname{Re}(\tilde{\nabla} f, \tilde{\nabla}(\tilde{\Delta} f)) \\
& +(\operatorname{Ric}+(n-2) / 2 \operatorname{Tor})(\tilde{\nabla} f, \tilde{\nabla} f)+i\left(D^{2} f\right)\left(X_{0},(\tilde{d} f)^{*}\right), \\
& \int_{M} i\left(D^{2} f\right)\left(X_{0},(\tilde{d} f)^{*}\right) d v=\frac{2}{n} \int_{M}\left\|\pi_{+} D^{2} f\right\|^{2}-\left\|\pi_{-} D^{2} f\right\|^{2}-\operatorname{Ric}(\tilde{\nabla} f, \tilde{\nabla} f) d v
\end{aligned}
$$

and

$$
\int_{M} i\left(D^{2} f\right)\left(X_{0},(\tilde{d} f)^{*}\right) d v=\int_{M}-\frac{4}{n}\left|\operatorname{tr}\left(\pi_{+} D^{2} f\right)\right|^{2}+\frac{1}{n}(\tilde{\Delta} f)^{2}+\operatorname{Tor}(\tilde{\nabla} f, \tilde{\nabla} f) d v
$$

where $\left\|\pi_{+} D^{2} f\right\|^{2},\left\|\pi_{-} D^{2} f\right\|^{2}, \operatorname{tr}\left(\pi_{+} D^{2} f\right)$ and $D^{2} f\left(X_{0},(\tilde{d} f)^{*}\right)$ are locally given by $\sum f_{\beta \bar{\alpha}} f_{\beta \bar{\alpha}}, \sum f_{\beta \alpha} f_{\bar{\beta} \bar{\alpha}}, \sum f_{\alpha \bar{\alpha}}$ and $\sum f_{\bar{\alpha}} f_{\alpha 0}-f_{\alpha} f_{\bar{\alpha} 0}$, respectively. 
If $f$ is a non-constant real-valued function so that $\tilde{\Delta} f=-\lambda_{1} f$, then $\int_{M} f d v=0$ and

$$
\lambda_{1} \int_{M}|f|^{2} d v=-\int_{M}(f, \tilde{\Delta} f) d v=2 \int_{M}|\tilde{\nabla} f|^{2} d v .
$$

Consequently, for any $c \in[0,1],(1-c) \times(2.3)+c \times(2.4)$ gives

$$
\begin{aligned}
\int_{M} i\left(D^{2} f\right)\left(X_{0},(\tilde{d} f)^{*}\right) d v \\
=\int_{M}\left[\frac{2(1-c)}{n}\left\|\pi_{+} D^{2} f\right\|^{2}-\frac{4 c}{n}\left|\operatorname{tr}\left(\pi_{+} D^{2} f\right)\right|^{2}-\frac{2(1-c)}{n}\left\|\pi_{-} D^{2} f\right\|^{2}\right. \\
\left.\quad+\frac{2 \lambda_{1} c}{n}|\tilde{\nabla} f|^{2}-\frac{2(1-c)}{n} \operatorname{Ric}(\tilde{\nabla} f, \tilde{\nabla} f)+c \operatorname{Tor}(\tilde{\nabla} f, \tilde{\nabla} f)\right] d v .
\end{aligned}
$$

By the Cauchy-Schwarz inequality,

$$
\left\|\pi_{+} D^{2} f\right\|^{2} \geq \frac{1}{n}\left|\operatorname{tr}\left(\pi_{+} D^{2} f\right)\right|^{2} .
$$

Then

$$
\begin{aligned}
0= & \int_{M} \frac{1}{2} \tilde{\Delta}|\tilde{\nabla} f|^{2} d v \\
= & \int_{M}\left[\left\|\pi_{+} D^{2} f\right\|^{2}+\left\|\pi_{-} D^{2} f\right\|^{2}-\lambda_{1} \operatorname{Re}(\tilde{\nabla} f, \tilde{\nabla} f)\right) \\
& +\left(\operatorname{Ric}+\frac{(n-2)}{2} \operatorname{Tor}\right)(\tilde{\nabla} f, \tilde{\nabla} f) \\
& +\frac{2(1-c)}{n}\left\|\pi_{+} D^{2} f\right\|^{2}-\frac{4 c}{n}\left|\operatorname{tr}\left(\pi_{+} D^{2} f\right)\right|^{2}-\frac{2(1-c)}{n}\left\|\pi_{-} D^{2} f\right\|^{2} \\
& \left.\quad+\frac{2 \lambda_{1} c}{n}|\tilde{\nabla} f|^{2}-\frac{2(1-c)}{n} \operatorname{Ric}(\tilde{\nabla} f, \tilde{\nabla} f)+c \operatorname{Tor}(\tilde{\nabla} f, \tilde{\nabla} f)\right] d v \\
\geq & \int_{M}\left[\left(\frac{1}{n}+\frac{2(1-c)}{n^{2}}-\frac{4 c}{n}\right)\left|\operatorname{tr}\left(\pi_{+} D^{2} f\right)\right|^{2}+\left(1-\frac{2(1-c)}{n}\right)\left\|\pi_{-} D^{2} f\right\|^{2}\right. \\
& \left.-\lambda_{1}\left(1-\frac{2 c}{n}\right)|\tilde{\nabla} f|^{2}+\left(\left(1-\frac{2(1-c)}{n}\right) \operatorname{Ric}+\frac{(n-2+2 c)}{2} \operatorname{Tor}\right)(\tilde{\nabla} f, \tilde{\nabla} f)\right] d v .
\end{aligned}
$$

To get rid of the $\left|\operatorname{tr}\left(\pi_{+} D^{2} f\right)\right|^{2}$ term, we solve $\frac{1}{n}+\frac{2(1-c)}{n^{2}}-\frac{4 c}{n}=0$ for $c$ and let $c=\frac{n+2}{2(1+2 n)}$. For this choice of $c$, the above inequality becomes

$$
0 \geq \int_{M} \frac{2(n-1)}{1+2 n}\left[\left\|\pi_{-} D^{2} f\right\|^{2}-\frac{(n+1) \lambda_{1}}{n}|\tilde{\nabla} f|^{2}+\left(\operatorname{Ric}+\frac{n}{2} \operatorname{Tor}\right)(\tilde{\nabla} f, \tilde{\nabla} f)\right] d v .
$$

By the hypothesis, $\left(\right.$ Ric $+\frac{n}{2}$ Tor $)(\tilde{\nabla} f, \tilde{\nabla} f) \geq k_{0}|\tilde{\nabla} f|^{2}$. Hence

$$
0 \geq \int_{M} \frac{2(n-1)}{2 n+1}\left[\left\|\pi_{-} D^{2} f\right\|^{2}+\left(k_{0}-\frac{n+1}{n} \lambda_{1}\right)|\tilde{\nabla} f|^{2}\right] d v,
$$

which implies that for $n \geq 2, \frac{n+1}{n} \lambda_{1} \geq k_{0}$. Therefore, we have proved Theorem 1.1 . 


\section{Proof of Theorem 1.2}

For $n=1$, we need more on the pseudo-Hermitian geometry of $M$. First we compute the curvature 2 -form $\Omega_{1}^{1}$ in (1.9) to get $\lambda_{1 \overline{1}}$ in (1.10) explicitly. The result is

$$
\Omega_{1}^{1}=d \omega_{1}^{1}=R_{1 \overline{1} 1 \overline{1}} \theta^{1} \wedge \theta^{\overline{1}}+W_{1 \overline{1} 1} \theta^{1} \wedge \theta-W_{\overline{1} 1 \overline{1}} \theta^{\overline{1}} \wedge \theta
$$

where

$$
\begin{gathered}
R_{1 \overline{1} 1 \overline{1}}=X_{1} \Gamma_{1 \overline{1}}^{1}-X_{\overline{1}} \Gamma_{11}^{1}+\Gamma_{11}^{1} \Gamma_{1 \overline{1}}^{1}-\Gamma_{1 \overline{1}}^{1} \Gamma_{\overline{1} 1}^{\overline{1}}+i \Gamma_{10}^{1}, \\
W_{1 \overline{1} 1}=X_{1} \Gamma_{10}^{1}-X_{0} \Gamma_{11}^{1}+\Gamma_{11}^{1} \Gamma_{10}^{1}-A_{11} \Gamma_{1 \overline{1}}^{1}
\end{gathered}
$$

and $W_{\overline{1} 1 \overline{1}}=\overline{W_{1 \overline{1} 1}}$. There is another curvature 2 -form $\Omega^{1}$ defined by

$$
\Omega^{1}=d \tau^{1}-\tau^{1} \wedge \omega_{1}^{1}
$$

Explicit computation gives

$$
\Omega^{1}=\left(X_{1} A_{\overline{11}}+2 A_{\overline{11}} \Gamma_{11}^{1}\right) \theta^{1} \wedge \theta^{\overline{1}}-\left|A_{\overline{11}}\right|^{2} \theta^{1} \wedge \theta+\left(-X_{0} A_{\overline{11}}-2 A_{\overline{11}} \Gamma_{10}^{1}\right) \theta^{\overline{1}} \wedge \theta
$$

It was shown in $\left[9\right.$ that the coefficient of $\theta^{1} \wedge \theta^{\bar{T}}$ in $\Omega^{1}$ is equal to $W_{\overline{1} 1 \overline{1}}$ in $\Omega_{1}^{1}$. Equating the two expressions of $W_{\overline{1} 1 \overline{1}}$, we get

$$
X_{1} A_{\overline{11}}=X_{0} \Gamma_{1 \overline{1}}^{1}-X_{\overline{1}} \Gamma_{10}^{1}+\Gamma_{1 \overline{1}}^{1} \Gamma_{\overline{1} 0}^{\overline{1}}-A_{\overline{11}} \Gamma_{11}^{1} .
$$

With this extra information, we will be able to prove the following lemma. For convenience, we shall henceforth write $A=A_{\overline{11}}$.

Lemma 3.1. Let $\tilde{\Delta} f=-\lambda_{1} f$ and $f_{0}=X_{0} f$. Then

$$
\frac{1}{2} \int_{M} \tilde{\Delta} f_{0}^{2} d v=-\lambda_{1} \int_{M} f_{0}^{2} d v+2 \int_{M}\left|X_{1} f_{0}\right|^{2} d v-4 \operatorname{Re} \int_{M} A f_{1} X_{1} f_{0} d v
$$

(both sides being zero).

Proof.

$$
\begin{aligned}
\frac{1}{2} \tilde{\Delta}\left(f_{0}^{2}\right) & =\frac{1}{2}\left(\left(f_{0}^{2}\right)_{1 \overline{1}}+\left(f_{0}^{2}\right)_{\overline{1} 1}\right) \\
& =\frac{1}{2}\left[X_{\overline{1}}\left(f_{0}^{2}\right)_{1}-\Gamma_{1 \overline{1}}^{1}\left(f_{0}^{2}\right)_{1}+X_{1}\left(f_{0}^{2}\right)_{\overline{1}}-\Gamma_{\overline{1} 1}^{\bar{T}}\left(f_{0}^{2}\right)_{\overline{1}}\right] \\
& =X_{\overline{1}}\left(f_{0} X_{1} f_{0}\right)-\Gamma_{1 \overline{1}}^{1}\left(f_{0} X_{1} f_{0}\right)+X_{1}\left(f_{0} X_{\overline{1}} f_{0}\right)-\Gamma_{\overline{1} 1}^{\overline{1}}\left(f_{0} X_{\overline{1}} f_{0}\right) \\
& =2\left|X_{1} f_{0}\right|^{2}+2 f_{0} \operatorname{Re}\left(X_{\overline{1}} X_{1} f_{0}\right)-2 f_{0} \operatorname{Re}\left(\Gamma_{1 \overline{1}}^{1} X_{1} f_{0}\right) .
\end{aligned}
$$


Using the Lie bracket $\left[X_{0}, X_{1}\right]=\Gamma_{10}^{1} X_{1}-\bar{A} X_{\overline{1}},(1.15)$ and (1.7), we get

$$
\begin{aligned}
X_{\overline{1}} X_{1} f_{0}= & X_{\overline{1}} X_{0} X_{1} f+X_{\overline{1}}\left[X_{1}, X_{0}\right] f \\
= & X_{0} X_{\overline{1}} X_{1} f+\left[X_{\overline{1}}, X_{0}\right] X_{1} f+X_{\overline{1}}\left[X_{1}, X_{0}\right] f \\
= & X_{0}\left(f_{1 \overline{1}}+\Gamma_{1 \overline{1}}^{1} f_{1}\right)+\left(A X_{1}-\Gamma_{\overline{1} 0}^{\overline{1}} X_{\overline{1}}\right) X_{1} f+X_{\overline{1}}\left(\bar{A} X_{\overline{1}}-\Gamma_{10}^{1} X_{1}\right) f \\
= & X_{0} f_{1 \overline{1}}+X_{0}\left(\Gamma_{1 \overline{1}}^{1}\right) f_{1}+\Gamma_{1 \overline{1}}^{1} X_{0} f_{1}+\left(A X_{1} X_{1}+\bar{A} X_{\overline{1}} X_{\overline{1}}\right) f \\
& -\Gamma_{\overline{1} 0}^{\overline{1}} X_{\overline{1}} X_{1} f+X_{\overline{1}}(\bar{A}) X_{\overline{1}} f-X_{\overline{1}}\left(\Gamma_{10}^{1}\right) X_{1} f-\Gamma_{10}^{1} X_{\overline{1}} X_{1} f \\
= & X_{0} f_{1 \overline{1}}+X_{0}\left(\Gamma_{1 \overline{1}}^{1}\right) f_{1}+\Gamma_{1 \overline{1}}^{1} X_{1} f_{0}+\Gamma_{1 \overline{1}}^{1}\left(-\bar{A} X_{\overline{1}}+\Gamma_{10}^{1} X_{1}\right) f \\
& +\left(A X_{1} X_{1}+\bar{A} X_{\overline{1}} X_{\overline{1}}\right) f+X_{\overline{1}}(\bar{A}) X_{\overline{1}} f-X_{\overline{1}}\left(\Gamma_{10}^{1}\right) X_{1} f \\
= & X_{0} f_{1 \overline{1}}+\left[X_{0}\left(\Gamma_{1 \overline{1}}^{1}\right)-X_{\overline{1}}\left(\Gamma_{10}^{1}\right)+\Gamma_{1 \overline{1}}^{1} \Gamma_{10}^{1}\right] f_{1}+\left(X_{\overline{1}}(\bar{A})-\Gamma_{1 \overline{1}}^{1} \bar{A}\right) f_{\overline{1}} \\
& +\left(A X_{1} X_{1}+\bar{A} X_{\overline{1}} X_{\overline{1}}\right) f+\Gamma_{1 \overline{1}}^{1} X_{1} f_{0} .
\end{aligned}
$$

Thus,

$$
\begin{aligned}
2 \operatorname{Re} X_{\overline{1}} X_{1} f_{0}= & X_{0}\left(f_{1 \overline{1}}+f_{\overline{1} 1}\right)+2 \operatorname{Re}\left[X_{0}\left(\Gamma_{1 \overline{1}}^{1}\right)-X_{\overline{1}}\left(\Gamma_{10}^{1}\right)+\Gamma_{1 \overline{1}}^{1} \Gamma_{10}^{1}+X_{1} A-A \Gamma_{\overline{1} 1}^{\overline{1}}\right] f_{1} \\
& +2\left(A X_{1} X_{1}+\bar{A} X_{\overline{1}} X_{\overline{1}}\right) f+2 \operatorname{Re} \Gamma_{1 \overline{1}}^{1} X_{1} f_{0} .
\end{aligned}
$$

Therefore,

$$
\begin{aligned}
\frac{1}{2} \tilde{\Delta} f_{0}^{2}= & 2\left|X_{1} f_{0}\right|^{2}-2 f_{0} \operatorname{Re}\left(\Gamma_{1 \overline{1}}^{1} X_{1} f_{0}\right) \\
& +f_{0}\left[X_{0}\left(f_{1 \overline{1}}+f_{\overline{1} 1}\right)+2 \operatorname{Re}\left[X_{0}\left(\Gamma_{1 \overline{1}}^{1}\right)-X_{\overline{1}}\left(\Gamma_{10}^{1}\right)\right.\right. \\
& \left.+\Gamma_{1 \overline{1}}^{1} \Gamma_{10}^{1}+X_{1} A-A \Gamma_{\overline{1} 1}^{\overline{1}}\right] f_{1} \\
& \left.+2\left(A X_{1} X_{1}+\bar{A} X_{\overline{1}} X_{\overline{1}}\right) f+2 \operatorname{Re} \Gamma_{1 \overline{1}}^{1} X_{1} f_{0}\right] \\
= & 2\left|X_{1} f_{0}\right|^{2}+2 f_{0}\left(A X_{1} X_{1}+\bar{A} X_{\overline{1}} X_{\overline{1}}\right) f \\
& +f_{0} X_{0} \tilde{\Delta} f+2 f_{0} \operatorname{Re}\left[X_{0}\left(\Gamma_{1 \overline{1}}^{1}\right)-X_{\overline{1}}\left(\Gamma_{10}^{1}\right)+\Gamma_{1 \overline{1}}^{1} \Gamma_{10}^{1}+X_{1} A-A \Gamma_{\overline{1} 1}^{\overline{1}}\right] f_{1} .
\end{aligned}
$$

Using $X_{\overline{1}}^{*}=-X_{1}+\Gamma_{\overline{1} 1}^{\overline{1}}$, we get

$$
\begin{aligned}
& 2 \int_{M} f_{0} A X_{1} X_{1} f d v \\
& =2 \int_{M} X_{\overline{1}}^{*}\left(f_{0} A\right) f_{1} d v \\
& =-2 \int_{M} X_{1}\left(f_{0} A\right) f_{1} d v+2 \int_{M} \Gamma_{\overline{1} 1}^{\overline{1}} f_{0} A d v \\
& =-2 \int_{M} X_{1}(A) f_{1} f_{0} d v-2 \int_{M} A f_{1} X_{1} f_{0} d v+2 \int_{M} \Gamma_{\overline{1} 1}^{\bar{T}} A f_{0} f_{1} d v .
\end{aligned}
$$


Then, by (3.4) with $A=A_{\overline{11}}$,

$$
\begin{gathered}
2 \int_{M} f_{0} A X_{1} X_{1} f d v+\int_{M}\left(\Gamma_{1 \overline{1}}^{1} \Gamma_{10}^{1}-X_{\overline{1}}\left(\Gamma_{10}^{1}\right)+X_{0}\left(\Gamma_{1 \overline{1}}^{1}\right)+X_{1} A-A \Gamma_{\overline{1} 1}^{\overline{1}}\right) f_{1} f_{0} d v \\
=-2 \int_{M} A f_{1} X_{1} f_{0} d v+\int_{M}\left(\Gamma_{1 \overline{1}}^{1} \Gamma_{10}^{1}-X_{\overline{1}}\left(\Gamma_{10}^{1}\right)\right. \\
\left.\quad+X_{0}\left(\Gamma_{1 \overline{\mathrm{T}}}^{1}\right)-X_{1} A+A \Gamma_{\overline{1} 1}^{\overline{1}}\right) f_{1} f_{0} d v \\
=-2 \int_{M} A f_{1} X_{1} f_{0} d v .
\end{gathered}
$$

Therefore,

$$
\int_{M} \frac{1}{2} \tilde{\Delta} f_{0}^{2} d v=2 \int_{M}\left|X_{1} f_{0}\right|^{2} d v-\lambda_{1} \int_{M}\left(f_{0}\right)^{2} d v-4 \operatorname{Re} \int_{M} A f_{1} X_{1} f_{0} d v
$$

and the proof of the lemma is complete.

Notice that

$$
\begin{aligned}
i\left(f_{\overline{\mathrm{T}}} f_{10}-f_{1} f_{\overline{\mathrm{T}} 0}\right) & \\
= & i f_{\overline{\mathrm{T}}}\left(X_{0} f_{1}-\Gamma_{10}^{1} f_{1}\right)-i f_{1}\left(X_{0} f_{\overline{1}}-\Gamma_{\overline{1} 0}^{\overline{1}} f_{\overline{1}}\right) \\
= & i f_{\overline{1}}\left(X_{1} f_{0}+\left(\Gamma_{10}^{1} f_{1}-\bar{A} f_{\overline{1}}\right)-\Gamma_{10}^{1} f_{1}\right) \\
& \quad-i f_{1}\left(X_{\overline{1}} f_{0}+\left(\Gamma_{\overline{1} 0}^{\overline{1}} f_{\overline{1}}-A f_{1}\right)-\Gamma_{\overline{1} 0}^{\overline{1}} f_{\overline{1}}\right) \\
= & i\left(f_{\overline{1}} X_{1} f_{0}-f_{1} X_{\overline{1}} f_{0}\right)+i\left(A f_{1}^{2}-\overline{A f}_{1}^{2}\right) \\
= & i\left(f_{\overline{1}} X_{1} f_{0}-f_{1} X_{\overline{1}} f_{0}\right)+\operatorname{Tor}(\tilde{\nabla} f, \tilde{\nabla} f),
\end{aligned}
$$

and

$$
X_{1}^{*}=-X_{\overline{1}}+\Gamma_{1 \overline{1}}^{1} \quad \text { and }\left[X_{1}, X_{\overline{1}}\right]=-i X_{0}-\Gamma_{1 \overline{1}}^{1} X_{1}+\Gamma_{\overline{1} 1}^{\overline{1}} X_{\overline{1}} .
$$

By (3.7)

$$
\begin{aligned}
\operatorname{Im} \int_{M} f_{\overline{1}} X_{1} f_{0} d v & =\operatorname{Im} \int_{M}\left(-X_{1} X_{\overline{1}} f+\Gamma_{\overline{1} 1}^{\overline{1}} f_{\bar{T}}\right) f_{0} d v \\
& =\operatorname{Im} \int_{M} \frac{i}{2} X_{0} f f_{0} d v \\
& =\frac{1}{2} \int_{M} f_{0}^{2} d v .
\end{aligned}
$$

Hence, for $n=1$, by (2.2), (3.6), (3.8) and the equation

$$
\begin{aligned}
\int_{M}\left|f_{1 \overline{\mathrm{I}}}\right|^{2} d v & =\int_{M}\left(\operatorname{Re} f_{1 \overline{1}}\right)^{2}+\left(\operatorname{Im} f_{1 \overline{\mathrm{I}}}\right)^{2} d v \\
& =\int_{M} \frac{1}{4}|\tilde{\Delta} f|^{2}+\frac{1}{4}\left|f_{0}\right|^{2} d v \\
& =\int_{M} \frac{\lambda_{1}}{2}|\tilde{\nabla} f|^{2}+\frac{1}{4}\left|f_{0}\right|^{2} d v
\end{aligned}
$$


we have

$$
\begin{aligned}
0= & \frac{1}{2} \int_{M} \tilde{\Delta}|\tilde{\nabla} f|^{2} d v \\
= & \int_{M}\left(\frac{\lambda_{1}}{2}|\tilde{\nabla} f|^{2}+\frac{1}{4}\left|f_{0}\right|^{2}\right)+\left|f_{11}\right|^{2}-\lambda_{1}|\tilde{\nabla} f|^{2} \\
& +\operatorname{Ric}(\tilde{\nabla} f, \tilde{\nabla} f)-\frac{1}{2} \operatorname{Tor}(\tilde{\nabla} f, \tilde{\nabla} f)+i\left(f_{\overline{\mathrm{T}}} f_{10}-f_{1} f_{\overline{\mathrm{I}} 0}\right) d v \\
= & \int_{M}\left(\frac{\lambda_{1}}{2}|\tilde{\nabla} f|^{2}+\frac{1}{4}\left|f_{0}\right|^{2}\right)+\left|f_{11}\right|^{2}-\lambda_{1}|\tilde{\nabla} f|^{2} \\
& +\left(\operatorname{Ric}+\frac{1}{2} \operatorname{Tor}\right)(\tilde{\nabla} f, \tilde{\nabla} f)+i\left(f_{\overline{1}} X_{1} f_{0}-f_{1} X_{\overline{1}} f_{0}\right) d v \\
= & \int_{M}\left(-\frac{1}{2} \lambda_{1}|\tilde{\nabla} f|^{2}+\frac{1}{4}\left|f_{0}\right|^{2}+\left|f_{11}\right|^{2}\right. \\
& +\left(\operatorname{Ric}+\frac{1}{2} \operatorname{Tor}\right)(\tilde{\nabla} f, \tilde{\nabla} f)-2 \operatorname{Im}\left(f_{\overline{1}} X_{1} f_{0}\right) d v \\
= & \int_{M}\left(-\frac{1}{2} \lambda_{1}|\tilde{\nabla} f|^{2}+\left|f_{11}\right|^{2}+\left(\operatorname{Ric}+\frac{1}{2} \operatorname{Tor}\right)(\tilde{\nabla} f, \tilde{\nabla} f)-\frac{3}{2} \operatorname{Im}\left(f_{\overline{1}} X_{1} f_{0}\right) d v .\right.
\end{aligned}
$$

By Lemma 3.1, where $\int_{M} \tilde{\Delta} f_{0}^{2} d v=0$, and (3.8), we have

$$
2 \int_{M}\left|X_{1} f_{0}\right|^{2}-4 \operatorname{Re} \int_{M} A f_{1} X_{1} f_{0} d v=\lambda_{1} \int_{M} f_{0}^{2} d v=2 \lambda_{1} \operatorname{Im} \int_{M} f_{\overline{1}} X_{1} f_{0} d v
$$

Thus,

$$
\begin{aligned}
\int_{M}\left|X_{1} f_{0}\right|^{2} & =2 \operatorname{Re} \int_{M} A f_{1} X_{1} f_{0} d v+\lambda_{1} \operatorname{Im} \int_{M} f_{\overline{1}} X_{1} f_{0} d v \\
& \leq 2 \operatorname{Re} \int_{M} A f_{1} X_{1} f_{0} d v+\lambda_{1}\left(\int_{M}\left|f_{\overline{1}}\right|^{2} d v\right)^{1 / 2}\left(\int_{M}\left|X_{1} f_{0}\right|^{2} d v\right)^{1 / 2} \\
& \leq 2 \operatorname{Re} \int_{M} A f_{1} X_{1} f_{0} d v+\frac{\lambda_{1}^{2}}{2} \int_{M}\left|f_{\overline{1}}\right|^{2} d v+\frac{1}{2} \int_{M}\left|X_{1} f_{0}\right|^{2} d v .
\end{aligned}
$$

Since

$$
\int_{M} X_{0}\left(A f_{1}^{2}\right) d v=0
$$

we have

$$
\int_{M} A f_{1} X_{0} f_{1} d v=-\frac{1}{2} \int_{M} X_{0}(A) f_{1}^{2} d v
$$

Thus,

$$
\begin{aligned}
\operatorname{Re} \int_{M} A f_{1} X_{1} f_{0} d v \\
\quad=\operatorname{Re} \int_{M} A f_{1}\left(X_{0} f_{1}+\bar{A} X_{\overline{1}} f-\Gamma_{10}^{1} f_{1}\right) d v \\
\quad=\operatorname{Re} \int_{M}\left(|A|^{2}\left|f_{1}\right|^{2}-\frac{1}{2} X_{0}(A) f_{1}^{2}-A \Gamma_{10}^{1} f_{1}^{2}\right) d v \\
\quad=\int_{M} \frac{1}{2} B^{2}(\tilde{\nabla} f, \tilde{\nabla} f) d v .
\end{aligned}
$$


If there is no confusion, we shall simply write

$$
B^{2}(\tilde{\nabla} f, \tilde{\nabla} f)=B^{2}\left|f_{1}\right|^{2} .
$$

(3.10)-(3.14) imply that

$$
\begin{aligned}
\int_{M}\left|X_{1} f_{0}\right|^{2} d v & \leq 4 \operatorname{Re} \int_{M} A f_{1} X_{1} f_{0} d v+\lambda_{1}^{2} \int_{M}\left|f_{\bar{T}}\right|^{2} d v \\
& \leq \int_{M}\left(2 B^{2}+\lambda_{1}^{2}\right)\left|f_{1}\right|^{2} d v .
\end{aligned}
$$

Therefore,

$$
\begin{aligned}
-\frac{3}{2} & \operatorname{Im} \int_{M} f_{\overline{1}} X_{1} f_{0} d v \\
& \geq-\frac{3}{2}\left(\int_{M}\left|f_{1}\right|^{2} d v\right)^{1 / 2}\left(\int_{M}\left|X_{1} f_{0}\right|^{2} d v\right)^{1 / 2} \\
& \geq-\frac{3 b}{4} \int_{M}\left|f_{1}\right|^{2} d v-\frac{3}{4 b} \int_{M}\left|X_{1} f_{0}\right|^{2} d v \\
& \geq-\frac{3 b}{4} \int_{M}\left|f_{1}\right|^{2} d v-\frac{3}{4 b} \int_{M}\left(2 B^{2}+\lambda_{1}^{2}\right)\left|f_{1}\right|^{2} d v
\end{aligned}
$$

For simplicity, we will use the notation

$$
\operatorname{Ric}(\tilde{\nabla} f, \tilde{\nabla} f)+\frac{1}{2} \operatorname{Tor}(\tilde{\nabla} f, \tilde{\nabla} f)=k\left|f_{1}\right|^{2} .
$$

Therefore,

$$
\begin{aligned}
0 & -\frac{1}{2} \lambda_{1} \int_{M}\left|f_{1}\right|^{2} d v+\int_{M} k\left|f_{1}\right|^{2} d v \\
& -\frac{3 b}{4} \int_{M}\left|f_{1}\right|^{2} d v-\frac{3}{4 b} \int_{M}\left(2 B^{2}+\lambda_{1}^{2}\right)\left|f_{1}\right|^{2} d v \\
= & -\lambda_{1} \int_{M}\left(\frac{1}{2}+\frac{3 \lambda_{1}}{4 b}\right)\left|f_{1}\right|^{2} d v+\int_{M}\left(k-\frac{3}{4} b-\frac{3 B^{2}}{2 b}\right)\left|f_{1}\right|^{2} d v
\end{aligned}
$$

Let $b=k_{0} / 2$. Then by (1.17) and (1.18),

$$
k-\frac{3 B^{2}}{2 b}=k-\frac{3 B^{2}}{k_{0}} \geq k_{0} .
$$

Thus,

$$
\lambda_{1} \geq \frac{\left(k_{0}-\frac{3 b}{4}\right)}{\frac{1}{2}+\frac{3 \lambda_{1}}{4 b}}=\frac{\left(4 k_{0}-3 b\right) b}{2 b+3 \lambda_{1}}=\frac{5 k_{0}^{2}}{4\left(k_{0}+3 \lambda_{1}\right)} .
$$

This holds if and only if $12 \lambda_{1}^{2}+4 k_{0} \lambda_{1} \geq 5 k_{0}^{2}$, i.e., $\left(2 \lambda_{1}-k_{0}\right)\left(6 \lambda_{1}+5 k_{0}\right) \geq 0$. Since $\lambda_{1}>0$, we have $6 \lambda_{1}+5 k_{0}>0$. Hence $\lambda_{1} \geq \frac{k_{0}}{2}$. Therefore, the proof of Theorem 1.2 is complete.

Finally, we remark that for $n=1,(1.2)$ and (1.5) reduce to $\theta=\theta^{\prime}, \theta^{1}=e^{i \alpha} \theta_{1}^{\prime}$ and $X_{0}^{\prime}=X_{0}, X_{1}^{\prime}=e^{i \alpha} X_{1}$ where $\alpha \in \mathbb{R}$. Under these transformations, it can be checked that the quantities considered in Theorem 1.2 also have intrinsic meaning even though they are expressed locally. 


\section{ACKNOWLEGEMENT}

The second author is partially supported by RGC Hong Kong. Part of this research was done while the first author visited the Mathematics Department at the Chineses University of Hong Hong; he thanks them for their hospitality.

\section{REFERENCES}

[1] M. S. Baouendi, P. Ebenfelt, and L. P. Rothschild, Real submanifolds in complex space and their mappings. Princeton Mathematical Series, 47, Princeton University Press, Princeton, NJ, 1999. MR 2000b:32066

[2] P. H. Bérard, From vanishing theorems to estimating theorems: the Bochner technique revisited, Bull. Amer. Math. Soc., 19 (1988), 371-406. MR 89i:58152

[3] J. P. Bourguignon, The "magic" of Weitzenböck formulas, Variational methods (Paris, 1988), 251-271, Progr. Nonlinear Differential Equations Appl., 4, Birkhäuser, Boston, MA, 1990. MR 94a:58181

[4] I. Chavel, Eigenvalues in Riemannian Geometry, Academic Press, Inc., New York, London, Toronto, Tokyo, 1984. MR 86g:58140

[5] Allan Greenleaf, The first eigenvalue of a sub-Laplacian on a pseudo-Hermitian manifold, Comm. Partial Differential Equations, 10(2) (1985), 191-217. MR 86f:58157

[6] P. Li, Lecture Notes on Geometric Analysis, Lecture Notes Series, No. 6, RIM, Global Analysis Research Center, Seoul National Univ., Korea (1993).

[7] N. Tanaka, A differential geometric study on strongly pseudo-convex manifolds, Kinokuniya Book-Store, Tokyo, 1975. MR 53:3361

[8] D.-C. Chang and S.-Y. Li, A Riemann zeta function associated to the sub-Laplacian on the unit sphere in $\mathbb{C}^{n}$, J. Anal. Math., 86 (2002), 25-48.

[9] S. Webster, Pseudo-Hermitian structures on a real hypersurface, J. Differential Geom., 13 (1978), 25-41. MR 80e:32015

[10] S. Webster, A remark on the Chern-Moser tensor, Special issue for S. S. Chern, Houston J. Math., 28 (2002), 433-435. MR 2003d:32047

[11] S. Webster, On the pseudo-conformal geometry of a Kähler manifold, Math. Z., 157 (1977), 265-270. MR 57:16666

[12] Wu, Hung Hsi, The Bochner technique in differential geometry, Math. Rep., 3 (1988), No. 2, i-xii, 289-538. MR 91h:58031

Department of Mathematics, University of California, Irvine, California 92697-3875

E-mail address: sli@math.uci.edu

Department of Mathematics, Lady Shaw Building, The Chinese University of Hong Kong, Shatin, N. T., Hong Kong

E-mail address: hsluk@math.cuhk.edu.hk 